\title{
Heuristic and exact solutions to the inverse power index problem for small voting bodies
}

\author{
Sascha Kurz and Stefan Napel \\ University of Bayreuth, 95440 Bayreuth, Germany \\ \{sascha.kurz,stefan.napel\}@uni-bayreuth.de
}

\begin{abstract}
Power indices are mappings that quantify the influence of the members of a voting body on collective decisions a priori. Their nonlinearity and discontinuity makes it difficult to compute inverse images, i.e., to determine a voting system which induces a power distribution as close as possible to a desired one. This paper considers approximations and exact solutions to this inverse problem for the Penrose-Banzhaf index, which are obtained by enumeration and integer linear programming techniques. They are compared to the results of three simple solution heuristics. The heuristics perform well in absolute terms but can be improved upon very considerably in relative terms. The findings complement known asymptotic results for large voting bodies and may improve termination criteria for local search algorithms.
\end{abstract}

Keywords electoral systems; simple games; weighted voting games; square root rule; Penrose limit theorem; Penrose-Banzhaf index; institutional design

Mathematics Subject Classification (2010) 91B12, 91A12, 90C10

\section{Introduction}

Collective decision rules and, in particular, heterogeneous voting weights for members of a committee, council, or shareholder meeting translate into influence on collective decisions in a nonlinear and even discontinuous fashion. This can be seen, for instance, by considering a decision quota of $q=50 \%$ and players $i \in N=\{1,2,3\}$ whose voting weights are given by either the vector (i) $w=\left(w_{1}, w_{2}, w_{3}\right)=$ $(33 . \overline{3}, 33 . \overline{3}, 33 . \overline{3})$, (ii) $w^{\prime}=(50-\varepsilon, 48+\varepsilon, 2)$, or (iii) $w^{\prime \prime}=(50+\varepsilon, 48-\varepsilon, 2)$ for small $\varepsilon>0$. The major weight change from $w$ to $w^{\prime}$ does not affect possibilities to form a winning coalition at all, where coalition $S \subseteq N$ is called winning if the cumulative weight of its members exceeds the quota. Namely, $S$ is winning if and only if $|S| \geq 2$. By symmetry, the distribution of influence can a priori be expected to equal $\left(\frac{1}{3}, \frac{1}{3}, \frac{1}{3}\right)$ for either of the voting systems described by $(q ; w)$ and $\left(q ; w^{\prime}\right)$. The minor change from $w^{\prime}$ to $w^{\prime \prime}$, in contrast, renders voter 1 a dictator with associated power distribution $(1,0,0)$.

Social scientists, philosophers and mathematicians have investigated various voting power indices which try to quantify the a priori distribution of influence on committee decisions. The Shapley-Shubik index (Shapley and Shubik 1954) and the Penrose-Banzhaf index (PBI) (Penrose 1946; Banzhaf 1965) are most prominent, but by far not the only ones. ${ }^{1}$ They help researchers clarify the non-trivial a priori power implications of different voting weight assignments to a wider audience. The combinatorial nature of weighted voting systems can, still, mislead the general public's intuition and even that of political practitioners. For instance, it was apparently not noted that the voting weights of the original six members of the European Economic Community, in use from 1958 to 1973, rendered Luxembourg a null player whenever the EEC Council applied its qualified majority rule. The public discussion very heated in, but not restricted to, Poland and Germany - in the wake of the $2007 \mathrm{EU}$ summit at

\footnotetext{
${ }^{1}$ See Felsenthal and Machover (1998) or Laruelle and Valenciano (2008) for overviews.
} 
which new voting rules for the EU Council were agreed reflected persistent confusion between voting weights and power.

Even to specialists, the discrete nature of voting rules still poses challenges. This is true, in particular, for the optimal design of a voting system. Certain normative desiderata, such as the equal representation of bottom-tier voters in a two-tier voting system, often call for a specific distribution of voting power. It is then a non-trivial exercise to find a deterministic voting rule that comes as close as possible to inducing the desired a priori power distribution. ${ }^{2}$ Simple gradient-like search algorithms, such as the ones considered by Leech $(2002,2003)$, Aziz et al. (2007), or Fatima et al. (2008), deliver excellent results for many instances of this so-called inverse power index problem but have never been evaluated formally. One can neither rule out that only a local minimum of the distance between the desired and the induced power vector has been identified. Nor are bounds known on the possible gap to a globally optimal voting rule. The latter might involve the intersection of several one-dimensional $(q ; w)$-rules and, therefore, need not even be a feasible result of the applied search algorithm.

Motivated by qualified majority voting in the EU, Słomczyński and Życzkowski $(2006,2007)$ have identified an elegant way to approximately solve the inverse problem for moderately big $n$ if the decision quota $q$ is a free parameter. ${ }^{3}$ Their heuristic suggestion is particularly appealing because it avoids discrepancies between voting weight and power. Namely, approximate proportionality between the normalized weight vector $w=\left(w_{1}, \ldots, w_{n}\right)$ and the induced PBI $B(q ; w)$ is achieved when the quota is set to $q^{*}=\frac{1}{2}\left(1+\sqrt{\sum w_{i}^{2}}\right)$. A desired power vector $\beta$ can hence approximately be induced simply by choosing $w=\beta$ and then calculating $q^{*}$. Because the rule $\left(q^{*} ; w\right)$ is simple and minimizes possible confusion between voting weight and power, it has motivated the prominent "Jagiellonian Compromise" (also known as double square root voting system) in the discussion of future voting rules for the EU Council (see, e.g., Kirsch et al. 2007).

Whether the decision quota $q$ is a free parameter, so that Słomczyński and Życzkowski's optimal $q^{*}$ indeed can be chosen, or not, depends on the application at hand. Even if it can, the lack of bounds on how well the $\left(q^{*} ; \beta\right)$-heuristic performs relative to the respective globally optimal solution to the inverse problem provides motivation for further research. Knowing more about the quality of the $\left(q^{*} ; \beta\right)$-heuristic is especially important for situations in which the heuristic can be expected to perform rather badly. The derivation of $q^{*}$ is based on a continuous approximation of the fundamentally discrete distribution of the cumulative weight of a random coalition. Its use is problematic when this approximation is inaccurate. This pertains especially - but not exclusively - to "small" voting bodies.

For a given number $n$ of players, the set of different binary voting systems or simple games is finite. On the one hand, this finiteness implies that many desired power distributions cannot be approximated too well. Nontrivially, this remains true even for large $n$ : Alon and Edelman's (2010) results imply that there is a sequence of desired power distributions $\left\{\beta^{n}\right\}_{n=1,2, \ldots}$ which stays at least a constant positive distance away (in the $\|\cdot\|_{1}$-norm) from any Penrose-Banzhaf power distribution. As shown in Kurz $(2012 \mathrm{~b})$, the desired power distributions $\beta^{n}=(0.75,0.25,0,0, \ldots, 0)$ have $\|\cdot\|_{1}$-distance of at least $\frac{14}{37}$ to the PBI of every complete simple game or weighted voting game for $2 \leq n \leq 16$ players.

On the other hand, the finiteness of the set of simple games suggests a trivial algorithm for solving the inverse problem: enumerate all systems $v$ with $n$ players, compute the respective power distribution - say, the PBI $B(v)$ - and then pick a game $v^{*}$ that induces the smallest achievable difference between ideal vector $\beta$ and $B(v)$ according to a suitable metric.

To this end, a growing literature has investigated methods for the efficient enumeration of voting systems (see, e.g., Keijzer 2009; Keijzer et al. 2010; Kurz 2012a). But, up to now, even the number of complete simple games (and also of weighted voting games) is unknown for $n>9$. So enumeration works only for voting bodies with few members. Exact solutions to the inverse problem can, fortunately, also be obtained for somewhat larger $n$ by integer linear programming (ILP) techniques. Such an

\footnotetext{
${ }^{2}$ Non-deterministic rules such as random dictatorship or random quota rules (Dubey and Shapley 1979, sec. 5) can easily solve the problem, but are generally not regarded as satisfactory.

${ }^{3}$ For very big $n$, except in rather pathological cases, the distinctions between voting weight and voting power become negligible. Limit results for $n \rightarrow \infty$ which render the inverse problem trivial date back to the seminal work by Lionel S. Penrose (1946, 1952), and have rigorously been investigated by Lindner and Machover (2004), Chang et al. (2006), and Lindner and Owen (2007).
} 
approach was recently presented in Kurz (2012b). It stands in the tradition of earlier applications of ILP to electoral systems, as discussed in Grilli di Cortona et al. (1999), Pennisi et al. (2007) or Ricca et al. (2012).

This paper draws on both methods - complete enumeration and ILP - as well as standard local search algorithms in order to evaluate the accuracy of three heuristic solutions to the inverse problem for the PBI. The first heuristic simply combines $w=\beta$ with $q^{\circ}=50 \%$; the second combines it with the "optimal quota" $q^{*}$ derived by Słomczyński and Życzkowski (2007); the third uses $\bar{q}=\frac{1}{2}+1 / \sqrt{\pi n}$. The latter quota is the average of $q^{*}$ computed over a set of $\beta$-vectors which is of particular interest for the egalitarian design of two-tier voting systems (Słomczyński and Życzkowski 2011).

We compute differences between the respective heuristic solution and the exact solution for three different metrics and a comprehensive grid of target vectors with up to $n=7$ voters. We also study approximations of the exact solutions for a large sample of grid points for $7<n \leq 15$ as well as selected real-world examples based on the so-called Penrose square root rule and EU population figures. The results allow the estimation of bounds (and termination criteria) for the accuracy of a candidate solution which has been obtained by conventional local search methods. This may be useful in applications where a specific voting power distribution is sought for a moderate number of council delegates, committee members, or business shareholders. We also analyze the significant magnitude of error that the mentioned heuristics can produce even for large $n$ in pathological cases.

In the following Section 2 we introduce binary voting systems and their basic properties. The inverse power index problem is formalized in Section 3, along with a brief discussion of worst-case bounds. In Section 4 we present the design of our comparative investigation. The corresponding results are the topic of Section 5. We conclude in Section 6.

\section{Binary voting systems}

We consider binary voting systems, i.e., each voter $i \in N:=\{1, \ldots, n\}$ casts a binary vote (e.g., "yes" or "no") and this determines a binary collective decision. Such a situation can mathematically be described by a Boolean function $v: 2^{N} \rightarrow\{0,1\}$, where $2^{N}$ denotes the set of subsets of $N$. A coalition $S \subseteq N$ can, e.g., be interpreted as the set of "yes"-voters for a particular (unspecified) proposal.

Definition $1 A$ simple game is a monotone Boolean function, i.e., a mapping $v: 2^{N} \rightarrow\{0,1\}$ with $v(S) \leq v(T)$ for all $S \subseteq T$, which additionally satisfies $v(\emptyset)=0, v(N)=1$.

Coalition $S \subseteq N$ is called winning if $v(S)=1$, and otherwise losing. $S \subseteq N$ is called a minimal winning coalition if it is winning and all proper subsets are losing. Similarly, a maximal losing coalition is losing and all its proper supersets are winning. A simple game is uniquely characterized by either its set of minimal winning coalitions or its set of maximal losing coalitions. For a proof and additional properties we refer the reader, e.g., to Taylor and Zwicker (1999).

As an example consider the simple game $v$ which is characterized by the set $\{\{1\},\{2,3\}\}$ of its minimal winning coalitions. Taking all supersets of the minimal winning coalitions yields $\{\{1\},\{1,2\},\{1,3\}$, $\{1,2,3\},\{2,3\}\}$ as the set of all winning coalitions. The remaining subsets are losing, with the set of the maximal losing coalitions given by $\{\{2\},\{3\}\}$.

The monotonicity imposed in Definition 1 is a very weak requirement. By introducing Isbell's desirability relation, i.e., $i \sqsupset j$ for two voters $i, j \in N$ if and only if $v(\{i\} \cup S \backslash\{j\}) \geq v(S)$ is satisfied for all $\{j\} \subseteq S \subseteq N \backslash\{i\}$ (see, e.g., Isbell 1956), one can define a particularly relevant subclass of the set of all simple games $\mathcal{S}$ :

Definition 2 A simple game $v$ is called complete (also called directed) if the binary relation $\sqsupset$ is a total preoder, i.e.

(1) $i \sqsupset i$ for all $i \in N$,

(2) $i \sqsupset j$ or $j \sqsupset i$ (including “ $i \sqsupset j$ and $j \sqsupset i$ ”) for all $i, j \in N$, and 
(3) $i \sqsupset j, j \sqsupset h$ implies $i \sqsupset h$ for all $i, j, h \in N$.

In our small example one can easily check that $1 \sqsupset 2 \sqsupset 3$. So $v \in \mathcal{C}$ where $\mathcal{C} \subset \mathcal{S}$ denotes the set of all complete simple games. Note that also $3 \sqsupset 2$, i.e., voters 2 and 3 are equally desirable.

Many binary voting systems which are used in practice belong to a further refinement of $\mathcal{S}$ :

Definition 3 A simple game $v$ is weighted if there exist non-negative weights $w_{i} \in \mathbb{R}_{\geq 0}$ and a positive quota $q \in \mathbb{R}_{>0}$ such that $v(S)=1$ if and only if $\sum_{i \in S} w_{i} \geq q$.

A weighted representation of our small example is given by $(q ; w)=[2 ; 2,1,1]$. We call weighted simple games weighted voting games and denote their collection by $\mathcal{W}$. Every weighted voting game is complete while not every complete simple game is weighted, i.e., $\mathcal{W} \subset \mathcal{C} \subset \mathcal{S}$. But each complete simple game and even each simple game can be represented as the intersection of $1 \leq k<\infty$ weighted voting games. The minimal number $k$ of weighted voting games is called the dimension of the simple game in question (see, e.g., Taylor and Zwicker 1999, Deuneko and Woeginger 2006). The presently known enumeration results for the three considered classes of binary voting systems are summarized in Table 1 (up to isomorphisms). See, e.g., Kurz (2012a) for details.

\begin{tabular}{|l|rrrrrrrrr|}
\hline \multicolumn{1}{r|}{$n$} & 1 & 2 & 3 & 4 & 5 & \multicolumn{1}{c}{6} & \multicolumn{1}{c|}{7} & \multicolumn{1}{c|}{8} \\
\hline$\# \mathcal{S}$ & 1 & 3 & 8 & 28 & 208 & 16351 & $>4.7 \cdot 10^{8}$ & $>1.3 \cdot 10^{18}$ & $>2.7 \cdot 10^{36}$ \\
$\# \mathcal{C}$ & 1 & 3 & 8 & 25 & 117 & 1171 & 44313 & 16175188 & 284432730174 \\
$\# \mathcal{W}$ & 1 & 3 & 8 & 25 & 117 & 1111 & 29373 & 2730164 & 989913344 \\
\hline
\end{tabular}

Table 1: Number of distinct simple games, complete simple games, and weighted voting games

There are several equivalent representations of binary voting structures besides Boolean functions and lists of, e.g., minimum winning coalitions. Simple games can, for instance, be described as independent sets in a graph, and Carreras and Freixas (1996) have introduced a very efficient matrix parameterization of $\mathcal{C}$. Our computation algorithms will exploit yet another possibility. Namely, we use that voting systems can be represented as points of a polyhedron which have integer coordinates only: for each $S \subseteq N$ define $0 \leq x_{S} \leq 1$ and add the constraints $x_{\emptyset}=0, x_{N}=1$, and $x_{S} \leq x_{T}$ for all $\emptyset \subseteq S \subset T \subseteq N$. Each integer solution $x \in\{0,1\}^{2^{n}}$ of this system of linear inequalities is in bijection to a simple game (with $v(S)=x_{S}$ ). Complete simple games and weighted voting games are described by additional constraints and auxiliary variables for the weights. This allows the investigation of all three classes of voting games by ILP techniques.

\section{The inverse power index problem}

Power indices are mappings from a set of feasible voting structures, such as $\mathcal{S}$ or $\mathcal{W}$, to non-negative real vectors which are meant to quantify the influence of the members of a voting body on collective decisions. The inverse power index problem consists in finding a voting system, e.g., $(q ; w) \in \mathcal{W}$, which induces a power distribution as close as possible to a desired one. More formally, for a given number $n$ of voters, the general inverse power index problem involves a set $\Gamma$ of feasible voting structures for $n$ players, a power index $\phi: \Gamma \rightarrow \mathbb{R}_{\geq 0}^{n}$, a desired power distribution $\beta \in \mathbb{R}_{\geq 0}^{n}$ with $\sum_{i=1}^{n} \beta_{i}=1$, and a metric $d: \mathbb{R}^{n} \times \mathbb{R}^{n} \rightarrow \mathbb{R}_{\geq 0}$ which measures the deviation between two power vectors. Of course, $d(x, y)=\|x-y\|$ is a suitable choice for any vector norm $\|\cdot\|$. Given these ingredients the inverse power index problem amounts to finding a solution to the minimization problem

$$
\min _{v \in \Gamma} d(\phi(v), \beta) .
$$

In this paper, we consider the special instance of this problem where $\Gamma \in\{\mathcal{S}, \mathcal{C}, \mathcal{W}\}$. We include $\mathcal{S}$ and $\mathcal{C}$ because they are significantly larger domains for $n \geq 5$ (see Table 1 ) and some prominent 
real-world electoral systems fail to correspond to weighted voting games. Examples include the current voting rules (Treaty of Nice) and the future ones (Treaty of Lisbon) for the EU Council, which are 3and 2-dimensional, respectively. We take the (normalized) Penrose-Banzhaf index $B(v)$ as the voting power index of interest.

Definition 4 For a given $n$-player simple game $v$ the absolute Penrose-Banzhaf index $B_{i}^{\prime}(v)$ for player $i$ is defined as

$$
B_{i}^{\prime}(v)=\frac{1}{2^{n-1}} \cdot \sum_{\emptyset \subseteq S \subseteq N \backslash\{i\}} v(S \cup\{i\})-v(S) .
$$

The (normalized) Penrose-Banzhaf index (PBI) $B_{i}(v)$ for player $i$ is defined as

$$
B_{i}(v)=\frac{B_{i}^{\prime}(v)}{\sum_{j=1}^{n} B_{j}^{\prime}(v)} .
$$

Our distance computations will be based on the $\|\cdot\|_{1}$-norm (i.e., the sum of deviations between $B_{i}(v)$ and $\beta_{i}$ for all players $\left.i\right)$, the $\|\cdot\|_{\infty}$-norm (i.e., the maximum deviation), and a weighted version of the former. Section 4 will provide more details.

To the best of our knowledge, there exists only one (non-trivial) non-approximative result on how well the inverse problem can be solved for the PBI in the worst case. It is useful for later reference to rephrase this rather recent finding by Alon and Edelman (2010):

Theorem 1 (Alon-Edelman) Let $n>k$ be positive integers, let $\varepsilon<\frac{1}{k+1}$ be a positive real, and let $v$ be a simple game with $n$ voters. If $\sum_{i=k+1}^{n} B(v, i) \leq \varepsilon$, then there exists a simple game $v^{\prime}$ with $k$ voters such that

$$
\sum_{i=1}^{k}\left|B(v, i)-B\left(v^{\prime}, i\right)\right|+\sum_{i=k+1}^{n}|B(v, i)-0| \leq \frac{(2 k+1) \varepsilon}{1-(k+1) \varepsilon}+\varepsilon
$$

So given a "large" game $v$ with $n$ players in which $1-\varepsilon$ of the total PBI (normalized to one) is concentrated amongst $k<n$ "major" players, it is possible to ignore the $n-k$ "minor" players, i.e., compute the PBI in the smaller game $v^{\prime}$ amongst the major players only, and make an error (in the $\|\cdot\|_{1}$-norm) of no more than the stated bound. As an example, consider the power distribution $\beta^{n}=(0.75,0.25,0, \ldots, 0) \in \mathbb{R}_{\geq 0}^{n}$ for $n \geq 2$ and choose $k=2, \varepsilon=\frac{1}{18}$. Let $v$ be a simple game involving $n$ players with $\sum_{i=3}^{n} B_{i}(v) \leq \varepsilon=\frac{1}{18}$, From Theorem 1 we conclude the existence of a simple game $v^{\prime}$ involving $k=2$ players with $\left\|B(v)-B\left(v^{\prime}\right)\right\|_{1} \leq \frac{(2 k+1) \varepsilon}{1-(k+1) \varepsilon}+\varepsilon=\frac{7}{18}$ (with $B\left(v^{\prime}\right)$ extended naturally from $\mathbb{R}_{\geq 0}^{2}$ to $\mathbb{R}_{\geq 0}^{n}$ ). The $\|\cdot\|_{1}$-distance between $\beta^{2}$ and the three possible 2-player PBI vectors $B\left(v^{\prime}\right) \in\left\{(1,0),\left(\frac{1}{2}, \frac{1}{2}\right),(0,1)\right\}$ is at least $\frac{1}{2}$. We, therefore, have

$$
\left\|B(v)-\beta^{n}\right\|_{1} \geq\left\|B\left(v^{\prime}\right)-\beta^{2}\right\|_{1}-\left\|B(v)-B\left(v^{\prime}\right)\right\|_{1} \geq \frac{1}{2}-\frac{7}{18}=\frac{1}{9} .
$$

If otherwise $\sum_{i=3}^{n} B_{i}(v) \geq \varepsilon$ then $\left\|B(v)-\beta^{n}\right\|_{1} \geq\left(1-B_{1}(v)-B_{2}(v)\right)+\varepsilon \geq 2 \varepsilon=\frac{1}{9}$. Hence, $\beta^{n}$ cannot be approximated by the PBI of a simple game with an $\|\cdot\|_{1}$-error less than $\frac{1}{9}$. The latter is the sharpest possible bound obtainable from Theorem 1. It can be improved computationally to slightly more than $\frac{14}{37}$ for $n \leq 11$ on $\mathcal{S}$ and for $n \leq 16$ on $\mathcal{C}$ and $\mathcal{W}$ (see Kurz 2012b).

\section{Design of the computational investigation}

When the inverse problem arises in political applications of constitutional design, PBI vectors $\beta$ which are proportional to the square root of a population size vector $p$ play an elevated role. The reason is 
that - under the probabilistic assumptions which underlie the PBI - a binary voting system $v$ with $B(v)=\beta$ and

$$
\beta_{i}=\frac{\sqrt{p_{i}}}{\sum_{j=1}^{n} \sqrt{p_{j}}} .
$$

would equalize the voting power of citizens in a two-tier system in which $n$ delegates adopt the bottomtier majority opinion of their respective constituency $i \in\{1, \ldots, n\}$ and then cast a $w_{i}$-weighted vote in a top-tier assembly (e.g., the EU Council). See Penrose (1946), Felsenthal and Machover (1998), Kaniovski (2008) or Kurz et al. (2012) for details. We will consider this Penrose square root rule for varying $n$ and some historical population figures in order to select target vectors $\beta$ in our computations.

In principle, however, any vector in $\mathbb{R}_{>0}^{n}$ whose entries sum up to 1 might be a desired power distribution $\beta$. For instance, the partners of a non-profit $R \& D$ joint venture might have made relative financial contributions of $\left(\frac{1}{3}, \frac{1}{3}, \frac{1}{9}, \frac{1}{9}, \frac{1}{9}\right)$ and possibly want to align a priori voting power to this vector as well as possible. Ideally, for a given number $n$ of voters, one would like to compare the exact and heuristic solutions to the inverse problem for all possible normalized target vectors $\beta \in \Delta(n-1)$, where $\Delta(n-1)$ denotes the $n-1$-dimensional unit simplex. Because this is computationally infeasible, we complement our analysis of some politically motivated vectors by vectors $\beta$ from a finite grid on $\Delta(n-1)$. And we resort to approximations of the exact solution when $n$ is too large.

We will compare the (approximated) exact solution of the inverse problem on domain $\mathcal{S}, \mathcal{C}$, or $\mathcal{W}$ for a given desired PBI $\beta$ with three different heuristics. They stay in the class $\mathcal{W}$ of weighted voting games and have in common that voting weights are set equal to the desired voting power, i.e., $w=\beta$. They pick a distinct quota, and hence typically a different voting system $v \in \mathcal{W}$.

The first heuristic - referred to as the 50\%-heuristic - just chooses $q^{\circ}=\frac{1}{2}$. Simple majority is arguably the most common majority rule in practice. The $50 \%$-heuristic simply picks it and ignores the potentially large discrepancies between voting weight and voting power that can arise.

The second, more sophisticated heuristic has been suggested by Słomczyński and Życzkowski (2006, 2007). Their motivation was to implement PBI vectors proportional to the square root of population sizes in the European Union, but the heuristic applies to arbitrary target vectors. Namely, the $q^{*}$ heuristic selects the quota

$$
q^{*}=\frac{1}{2} \cdot\left(1+\sqrt{\sum_{i} w_{i}^{2}}\right)
$$

for an arbitrary $w=\beta \in \Delta(n-1)$. Słomczyński and Życzkowski (2007) derive this quota by considering the random weight $W$ which is accumulated if all coalitions $S \subseteq N$ are equiprobable, as the PBI's probabilistic justifications suppose. Equiprobability at the level of coalitions is equivalent to assuming that each voter $i \in\{1, \ldots, n\}$ joins the formed coalition independently of the others with probability $\frac{1}{2}$. The mean of $W$ hence is $\mu=\sum_{i=1}^{n} \frac{1}{2} w_{i}=\frac{1}{2}$ and its variance is $\sigma^{2}=\frac{1}{4} \sum_{i=1}^{n} w_{i}^{2}$. Being the sum of independent bounded random variables, $W$ is approximately normally distributed if $n$ is sufficiently large and each of the weights is sufficiently small. ${ }^{4}$ Assuming that this is the case and, therefore, that the discrete random variable $W$ can be replaced by the continuous one $\tilde{W}$, the inflection point of the corresponding normal density of $\tilde{W}$ is located at $q^{*}=\mu+\sigma$. Since the second derivative of $\tilde{W}$ 's density vanishes at $q^{*}$, one can approximate the density by a linear function with reasonably high accuracy. This linear approximation then allows to establish approximate proportionality of $B\left(q^{*} ; w\right)$ and $w$. We refer to Słomczyński and Życzkowski (2007) for details.

Our final heuristic, which we will refer to as the $\bar{q}$-heuristic, replaces $q^{*}$ by

$$
\bar{q}=\frac{1}{2}+\frac{1}{\sqrt{\pi n}} .
$$

This quota approximates the expected value of $q^{*}$ when $\beta$ is proportional to the component-wise square root of a population size vector $p=\left(p_{1}, \ldots, p_{n}\right)$ which is drawn from a flat Dirichlet distribution (see Słomczyński and Życzkowski 2011). The motivation for computing such an average is the following:

\footnotetext{
${ }^{4} \mathrm{~A}$ key technical requirement is $\max _{j} w_{j} \ll \sqrt{\sum_{i=1}^{n} w_{i}^{2}}$.
} 
even though the $q^{*}$-heuristic can approximate the Penrose square root rule (2) very transparently for a given population distribution $p$, frequent changes in the population would call not only for frequent changes of the heuristic's preferred voting weights $w$ but also of the quota $q^{*}$. That current voting weights in the EU already recur to population figures, which are updated on an annual basis, suggests that weight changes may be regarded as unproblematic. A varying decision threshold - perhaps $q=65 \%$ in one year, $q^{\prime}=61 \%$ in the next, then $q^{\prime \prime}=67 \%$, etc. - however seems politically less palatable. It may then make sense to average $q^{*}$ over a wide range of values for $w=\beta \propto \sqrt{p}$, and the $\bar{q}$-heuristic simply assumes that all population distributions $p \in \Delta(n-1)$ are equally likely. ${ }^{5}$ Because $\bar{q} \rightarrow \frac{1}{2}$ as $n \rightarrow \infty$, the $50 \%$-heuristic is the limit of the $\bar{q}$-heuristic and can be viewed as an approximation of it for not too small $n$.

Let us remark that investigations by Kurth (2008) have called attention to numerical problems when heuristics which involve irrational voting weights and quotas, as the $q^{*}$ or $\bar{q}$-heuristics commonly do, are implemented. Rounding after, e.g., 4 decimal places can result in voting systems which differ significantly from what was intended. Because it is impractical to deal with weights of a hundred decimal places or more, it is attractive to work with the underlying Boolean functions or integer points of a suitable polyhedron as long as possible, and to determine minimal integer weights $w$ and a quota $q$ which jointly represent a given $v \in \mathcal{W}$ when needed. ${ }^{6}$ We use this approach here whenever possible, and refer the interested reader to Freixas and Molinero (2009) or Kurz (2012a).

We calculate the globally optimal solution to the inverse problem for a given target PBI $\beta$ by complete enumeration of the elements in the respective class of binary voting systems for $n \leq 7$ (see Table 1). For larger $n$, we mostly focus on approximations of the exact solution. These are obtained either by a fast local search algorithm or, preferably, by ILP techniques. How the latter are used is explained in the Appendix. The implemented ILP-based algorithm in principle yields globally optimal solutions but is interrupted for computation time reasons when a desired precision has been reached. The key idea is to consider the integer polyhedron which contains all simple games whose PBI is less than a given factor $\alpha>0$ away from the desired vector $\beta$. If this polyhedron is empty, no such game exists and $\alpha$ needs to be raised. If not, $\alpha$ can be lowered. The minimal level of $\alpha$ or an approximation with desired precision, together with the corresponding voting systems, can thus be found by the bisection method.

In evaluating the quality of the mentioned heuristics, we consider distances to the desired power vector, $\beta$, and to the globally optimal one, $B\left(v^{*}\right)$, in three different metrics. The first one is the metric $d_{1}(x, y):=\|x-y\|_{1}=\sum_{i=1}^{n}\left|x_{i}-y_{i}\right|$ induced by the $\|\cdot\|_{1}$-norm, which is also considered in Theorem 1. The second is the metric induced by the $\|\cdot\|_{\infty}$-norm, i.e., $d_{\infty}(x, y):=\|x-y\|_{\infty}=\max _{i \in\{1, \ldots, n\}}\left|x_{i}-y_{i}\right|$. We refrain from also considering the Euclidean metric induced by the $\|\cdot\|_{2}$-norm, which has been considered, e.g., by Słomczyński and Życzkowski (2007). The reason is that this would turn the ILP formulation of the inverse power index problem into a binary non-linear programming one. This would be considerably harder to solve and add relatively little information because $\|x\|_{\infty} \leq\|x\|_{2} \leq \sqrt{n}\|x\|_{\infty}$ for all $x \in \mathbb{R}^{n}$.

More interesting, in our view, is a variation of $d_{1}$ which takes the Bernoulli model that underlies the PBI and Penrose's square root rule seriously. This model assumes that all bottom-tier voters in constituency $i \in\{1, \ldots, n\}$ cast a "yes" or "no" vote equiprobably and independently of all others. The probability for one of $p_{i}$ individual voters in constituency $i$ to be pivotal for the constituency's aggregate decision - i.e., to induce the $i$-delegate at the top-tier council to cast voting weight $w_{i}$ in favor of "yes" by individually voting "yes", and "no" by voting "no" - is approximately $\left.\sqrt{2 /\left(\pi p_{i}\right.}\right)$. The joint probability of a given voter being pivotal in his constituency $i$ and of this constituency being pivotal at the top tier is hence $\left.B_{i}(v) \cdot \sqrt{2 /\left(\pi p_{i}\right.}\right)$. This is why the square root PBI vector in equation (2) equalizes the indirect influence of citizens on collective decisions across constituencies. If

\footnotetext{
${ }^{5}$ The expected value of the $p$-specific optimal quotas $q^{*}(p)$ for a particular (e.g., Dirichlet) distribution of $p$, of course, need not coincide with the quota that is optimal when $p$ is treated as a random variable. Stochastic optimization techniques are likely to yield a somewhat better $q$-heuristic than the one suggested by Słomczyński and Życzkowski (2011).

${ }^{6} \mathrm{~A}$ minimal integer representation of a weighted voting game has the additional advantage that the PBI and other power indices can be computed most efficiently.
} 
one now weights any deviation between the probability for a given voter in constituency $i$ to be doubly pivotal and the egalitarian ideal of $\left.\beta_{i} \cdot \sqrt{2 /\left(\pi p_{i}\right.}\right)$ with $\beta_{i}=\sqrt{p_{i}} / \sum_{j=1}^{n} \sqrt{p_{j}}$ equally, then the total misrepresentation associated with the top-tier voting system $v$ amounts to

$$
\left.\sum_{i=1}^{n} p_{i} \cdot\left|\beta_{i}-B_{i}(v)\right| \cdot \sqrt{2 /\left(\pi p_{i}\right.}\right)=c \cdot \sum_{i=1}^{n} \sqrt{p_{i}} \cdot\left|\beta_{i}-B_{i}(v)\right|
$$

for $c>0$. Whenever the desired vector $\beta$ is derived from Penrose's square root rule and a vector $p$ which represents EU population data, we will, therefore, also consider the variation of metric $d_{1}$ which weights absolute deviations by the square root of relative population, i.e., study the metric ${ }^{7}$

$$
d_{1}^{\prime}(x, y):=\sum_{i=1}^{n} \sqrt{\frac{p_{i}}{\sum_{j=1}^{n} p_{j}}} \cdot\left|x_{i}-y_{i}\right|
$$

\section{Computational results}

In this section we present our numerical results. Subsection 5.1 considers the EU Council of Ministers as a prototype of a real-world weighted voting system. We then look at the entire space of possible power distributions for $n \leq 7$ and a random sample thereof in Subsection 5.2. In order to study analytically how deviations between heuristics and actual optimization depend on $n$, we investigate a particular parametric example in Subsection 5.3.

\subsection{Examples of real-world weighted voting systems}

We consider the (EEC or EC or) EU Council of Ministers in the years 1958, 1973, 1981, 1986, 1995, 2006 , and 2011 with respectively $n \in\{6,9,10,12,15,25,27\}$ members. The historical population data for $n \in\{6, \ldots, 15\}$ are taken from Felsenthal and Machover (1998, sec. 5.3), the data for $n \in\{25,27\}$ are official Eurostat figures downloaded on 19.01.2012. The desired power distribution $\beta$ is computed by Penrose's square root rule (see equation (2)).

In Tables 2-4 we compare the three considered heuristics under different metrics with the optimal solution of the inverse power index problem, where we distinguish $\mathcal{S}, \mathcal{C}$, and $\mathcal{W}$ as the set of feasible voting structures. Besides the absolute deviations measured in the respective metric we also report a relative measure: if the deviation of a certain heuristic is given by $\delta$ and that of the optimal solution $v^{*} \in \mathcal{S}$ is $\alpha$ - this is the unavoidable absolute "error" associated with the given instance of the inverse problem - then the tables report the avoidable error $(\delta-\alpha) / \alpha$ relative to global optimization in $\mathcal{S}$ (labeled $\mathcal{S}$-error in the tables). A value of 1 , e.g., means that the heuristic's approximation error is twice the unavoidable one. The "†"-symbol indicates that the stated value is not computationally proven to be optimal: for simple games and $n=9$, for instance, we stopped the ILP solution process after memory usage of $31 \mathrm{~GB}$ and 18461700 branch-and-bound nodes; for $n=10$, we interrupted after $301 \mathrm{~GB}$ and 16735508 nodes. Light figures for $\mathcal{S}$ or $\mathcal{C}$ represent lower bounds inferred from $\mathcal{W}$, and " $\infty$ " entries indicate deviations by factors greater than 500 .

Independently of the chosen metric, ${ }^{8}$ the tables show: (i) the approximation errors of the heuristics and the optimal solutions in $\mathcal{W}$ (and a fortiori in $\mathcal{C}$ and $\mathcal{S}$ ) tend to zero as $n$ increases; (ii) except for $n=9$, the $q^{*}$ - and the $\bar{q}$-heuristics perform noticeably better than the $50 \%$-heuristic; (iii) the $q^{*}$ and $\bar{q}$-heuristics produce comparable errors for $n \leq 15$ but differ significantly for $n>15$; (iv) the respective optimal weighted games $v^{* * *} \in \mathcal{W}$ yield deviations that are only moderately higher than those of $v^{*} \in \mathcal{S}$; and $(\mathrm{v})$ the relative errors of the heuristics compared to either $v^{*} \in \mathcal{S}$ or $v^{* *} \in \mathcal{W}$ are sizeable even for small $n \leq 15$ and become huge for $n>25$.

\footnotetext{
${ }^{7}$ Consideration of a similar variation of $d_{\infty}$ broadly confirms the comparisons based on $d_{1}, d_{1}^{\prime}$, and $d_{\infty}$.

${ }^{8}$ Note that the three metrics behave differently when, e.g., distance between $(1,0, \ldots, 0)$ and $\left(\frac{1}{n}, \ldots, \frac{1}{n}\right) \in \Delta(n-1)$ is considered for increasing $n$. Deviations should, therefore, be compared only within and not across tables.
} 


\begin{tabular}{|c|c|c|c|c|c|c|c|c|c|}
\hline \multirow{3}{*}{$\frac{n}{6}$} & \multirow{2}{*}{$\begin{array}{c}v^{*} \in \mathcal{S} \\
d_{1}\end{array}$} & \multirow{3}{*}{$\begin{array}{c}v^{* *} \in \mathcal{C} \\
d_{1} \\
0.051857\end{array}$} & \multirow{3}{*}{$\begin{array}{c}v^{* * *} \in \mathcal{W} \\
d_{1} \\
0.051857\end{array}$} & \multicolumn{2}{|c|}{$50 \%$-heuristic } & \multicolumn{2}{|c|}{$q^{*}$-heuristic } & \multicolumn{2}{|c|}{$\bar{q}$-heuristic } \\
\hline & & & & $d_{1}$ & -error & $d_{1}$ & -error & $d_{1}$ & -error \\
\hline & .051857 & & & 0.300398 & 4.79 & 0.091100 & 0.76 & 0.091100 & 0.76 \\
\hline 9 & & & & & 11. & & 10 & & 12 \\
\hline 10 & & & & 0. & 13. & 9 & 11 & 466 & 9. \\
\hline 12 & $14^{\dagger}$ & $37^{\dagger}$ & $\dagger$ & $0.02870 \mathrm{c}$ & 11.24 & 27 & 7 . & 27 & 7.4 \\
\hline 15 & 00476 & 76 & $0.000476^{\dagger}$ & 42 & 55.18 & 20 & 13.33 & 361 & 12.36 \\
\hline 25 & 0.000000 & 00 & $0.000000^{\dagger}$ & 0.019422 & $" \infty "$ & 0.000744 & $" \infty "$ & 0.003096 & $" \infty "$ \\
\hline 27 & 0.000000 & 0.000000 & $0.000000^{\dagger}$ & 0.018003 & $" \infty "$ & 0.000633 & $" \infty "$ & 0.002457 & $" \infty "$ \\
\hline
\end{tabular}

Table 2: Performance for Penrose square root targets in the $d_{1}$-metric (1958-2011 EU data)

The last observation is probably the most interesting: whenever one seeks an optimal solution of the inverse power index problem, all three heuristics are unsatisfactory from a pure operations research perspective. The heuristic solutions can be improved by very large factors, and this becomes more rather than less pronounced as $n$ grows. Of course, from an applied point of view the absolute approximation errors get so small for large $n$ that they may be regarded as negligible. But they may still be relevant. In order to see what a deviation at the 5 th decimal place means consider, e.g., the Penrose square root power distribution $\beta^{27}$ for the EU Council from 2011 and compare it to $\beta^{27 \prime}$ which would result if 50000 people moved from Germany to France or were mis-counted in the statistics. Then $\left\|\beta^{27}-\beta^{27 \prime}\right\|_{1} \approx 0.0000634$.

\begin{tabular}{|c|c|c|c|c|c|c|c|c|c|}
\hline \multirow{2}{*}{$\frac{n}{6}$} & $\begin{array}{c}v^{*} \in \mathcal{S} \\
d_{1}^{\prime}\end{array}$ & \multirow{2}{*}{$\begin{array}{c}v^{* *} \in \mathcal{C} \\
d_{1}^{\prime} \\
0.021487\end{array}$} & \multirow{2}{*}{$\begin{array}{c}v^{* * *} \in \mathcal{W} \\
d_{1}^{\prime} \\
0.021487\end{array}$} & \multicolumn{2}{|c|}{$\begin{array}{cc}50 \% \text {-heuristic } \\
d_{1}^{\prime} \quad \mathcal{S} \text {-error }\end{array}$} & \multicolumn{2}{|c|}{$\begin{array}{c}q^{*} \text {-heuristic } \\
d_{1}^{\prime} \quad \mathcal{S} \text {-error }\end{array}$} & \multicolumn{2}{|c|}{$\begin{array}{l}\bar{q} \text {-heuristic } \\
d_{1}^{\prime} \quad \mathcal{S} \text {-error }\end{array}$} \\
\hline & 0.018967 & & & \begin{tabular}{|l|}
0.110284 \\
\end{tabular} & 4.81 & 0.027 & 0.45 & 0.027465 & 0.45 \\
\hline 9 & 0 & & & & 9.00 & & 8.96 & & 8. \\
\hline 10 & $0.000803^{\dagger}$ & 0. & 0. & $0 . r^{2}$ & 10 & 0.007325 & 8. & 489 & $5.8>>$ \\
\hline 12 & $0.000309^{\dagger}$ & $0.001164^{\dagger}$ & $0.000810^{\dagger}$ & $0.00^{\prime}$ & 24.37 & 00400 & 11.96 & 0.004005 & 11.96 \\
\hline 15 & 0.000152 & 0.000152 & $0.000152^{\dagger}$ & 0.007790 & 50.26 & 0.00123 & $7 .(1$ & 0.001554 & 9.23 \\
\hline 25 & 0.000000 & 0.0 & $0.000000^{\dagger}$ & 0.004874 & $" \infty "$ & 0213 & $" \infty "$ & 0751 & $" \infty "$ \\
\hline 27 & & & $0.000000^{\dagger}$ & 0.004411 & $" \infty$ & 0.000176 & $" \infty "$ & 0.000578 & $" \infty "$ \\
\hline
\end{tabular}

Table 3: Performance for Penrose square root targets in the $d_{1}^{\prime}$-metric (1958-2011 EU data)

\begin{tabular}{|c|c|c|c|c|c|c|c|c|c|}
\hline$n$ & $\begin{array}{c}v^{*} \in \mathcal{S} \\
d_{\infty}\end{array}$ & $\begin{array}{c}v^{* *} \in \mathcal{C} \\
d_{\infty}\end{array}$ & $\begin{array}{c}v^{* * *} \in \mathcal{W} \\
d_{\infty}\end{array}$ & \multicolumn{2}{|c|}{$\begin{array}{c}50 \% \text {-heuristic } \\
d_{\infty} \quad \mathcal{S} \text {-error }\end{array}$} & \multicolumn{2}{|c|}{$\begin{array}{l}q^{*} \text {-heuristic } \\
d_{\infty} \quad \mathcal{S} \text {-error }\end{array}$} & \multicolumn{2}{|c|}{$\begin{array}{cc}\bar{q} \text {-heuristic } \\
d_{\infty} \quad \mathcal{S} \text {-error }\end{array}$} \\
\hline 6 & 0.014948 & 0.014948 & 0.014948 & 0.082758 & 4.54 & \begin{tabular}{|l|}
0.032728 \\
\end{tabular} & 1.19 & \begin{tabular}{|l}
0.032728 \\
\end{tabular} & 1.19 \\
\hline 9 & $0.001498^{\dagger}$ & 1840 & & 38 & 11.84 & 09 & 9.62 & 0.023179 & 14.47 \\
\hline 10 & $00575^{\dagger}$ & $1500^{\dagger}$ & $0.001960^{\dagger}$ & 74 & 19.13 & 16 & 9.98 & 9721 & 15.91 \\
\hline 12 & $0229^{\dagger}$ & $5880^{\dagger}$ & 0. & 40 & 33.67 & 56 & 24.13 & 56 & 13 \\
\hline 15 & & & & 3 & 88.74 & 8 & 26.24 & 02 & 17.21 \\
\hline 25 & 000 & 00 & $00^{\dagger}$ & 0.003834 & $" \infty "$ & 0.0 & $" \infty "$ & 0.000 & $" \infty "$ \\
\hline 27 & 0.000000 & 0.000000 & $0.000000^{\dagger}$ & 0.003434 & $" \infty "$ & 0.000156 & $" \infty "$ & 0.000277 & $" \infty "$ \\
\hline
\end{tabular}

Table 4: Performance for Penrose square root targets in the $d_{\infty}$-metric (1958-2011 EU data) 


\subsection{Finite grid of objective vectors}

Every vector in $\mathbb{R}_{\geq 0}^{n}$ whose entries sum to 1 can, in principle, be a desired power distribution in a specific context. We approximate this infinite space by a discrete grid. We impose $\beta_{1} \geq \beta_{2} \geq \ldots \geq \beta_{n}$ and let the desired power of the first $n-1$ voters be an integral multiple of $s=0.01 .^{9}$ So given $n$ and $s$, a finite set of desired power distributions arises, which we call grid points. ${ }^{10}$ Table 5 reports key statistics for the distribution of unavoidable deviations from the ideal vectors in the $d_{1}$ and $d_{\infty}$-metrics: its median, average, $10 \%, 5 \%$, and 1\%-percentile. The deviation figures are based on the respective exact solutions in $\mathcal{W}$ for $n \leq 7$ and approximations thereof for larger $n$. A number of grid points in parentheses indicates the size of the considered random sample whenever only a subset of all grid points could be dealt with. The deviation statistics in the corresponding row (in light color) involve a sample error in addition to the small error of using a conventional local search algorithm instead of global optimization in $\mathcal{W}$.

\begin{tabular}{|r|r|ccccc|ccccc|}
\hline & \#grid & \multicolumn{6}{|c|}{$d_{1}$-metric } & \multicolumn{5}{c|}{$d_{\infty}$-metric } \\
$n$ & points & med. & av. & $10 \%$ & $5 \%$ & $1 \%$ & med. & av. & $10 \%$ & $5 \%$ & $1 \%$ \\
\hline 2 & 51 & 0.240 & 0.245 & 0.040 & 0.020 & 0.000 & 0.120 & 0.123 & 0.020 & 0.010 & 0.000 \\
3 & 858 & 0.240 & 0.229 & 0.100 & 0.073 & 0.027 & 0.120 & 0.115 & 0.050 & 0.037 & 0.013 \\
4 & 7519 & 0.160 & 0.162 & 0.087 & 0.067 & 0.040 & 0.070 & 0.071 & 0.040 & 0.030 & 0.017 \\
5 & 41334 & 0.100 & 0.112 & 0.060 & 0.052 & 0.033 & 0.040 & 0.042 & 0.023 & 0.020 & 0.011 \\
6 & 160668 & 0.066 & 0.077 & 0.040 & 0.036 & 0.021 & 0.022 & 0.025 & 0.013 & 0.011 & 0.008 \\
7 & 477213 & 0.041 & 0.051 & 0.026 & 0.021 & 0.017 & 0.012 & 0.015 & 0.008 & 0.007 & 0.005 \\
8 & $(10000)$ & 0.042 & 0.046 & 0.026 & 0.023 & 0.017 & 0.012 & 0.014 & 0.007 & 0.006 & 0.005 \\
9 & $(10000)$ & 0.032 & 0.035 & 0.020 & 0.018 & 0.014 & 0.008 & 0.010 & 0.005 & 0.004 & 0.003 \\
10 & $(10000)$ & 0.025 & 0.027 & 0.016 & 0.014 & 0.011 & 0.006 & 0.007 & 0.004 & 0.003 & 0.003 \\
11 & $(10000)$ & 0.020 & 0.022 & 0.013 & 0.012 & 0.009 & 0.005 & 0.005 & 0.003 & 0.002 & 0.002 \\
12 & $(10000)$ & 0.016 & 0.018 & 0.011 & 0.009 & 0.007 & 0.004 & 0.004 & 0.002 & 0.002 & 0.001 \\
13 & $(10000)$ & 0.013 & 0.014 & 0.008 & 0.007 & 0.006 & 0.003 & 0.003 & 0.002 & 0.001 & 0.001 \\
14 & $(10000)$ & 0.010 & 0.011 & 0.006 & 0.006 & 0.004 & 0.002 & 0.002 & 0.001 & 0.001 & 0.001 \\
15 & $(10000)$ & 0.008 & 0.009 & 0.005 & 0.004 & 0.003 & 0.002 & 0.002 & 0.001 & 0.001 & 0.001 \\
\hline
\end{tabular}

Table 5: Distribution of unavoidable absolute deviations $d_{1}\left(\beta, B\left(v^{*}\right)\right.$ and $d_{\infty}\left(\beta, B\left(v^{*}\right)\right.$

Tables 6-8 report analogous statistics for the distribution of absolute distances for the three heuristics (considering each grid point for $n \leq 11$ and samples from the respective grid for $n>11$ ). A comparison of the respective deviation statistics with those in Table 5 confirm the observations that were made for the very specific grid points derived from Penrose's square root rule in Section 5.1: the average and each reported percentile of the avoidable deviations decrease in $n$. They can be regarded as small in absolute terms, but they are sizeable in relative terms. Again the 50\%-heuristic is clearly outperformed (in the sense of first order stochastic dominance) by the $q^{*}$ and $\bar{q}$-heuristics for $n \geq 3$.

\subsection{Analytical example}

That relative deviations between the considered heuristics and globally optimal solutions need not disappear for $n \rightarrow \infty$ can be seen very transparently by considering the desired power distribution

$$
\beta^{n}:=\frac{1}{2 n-1}(\overbrace{2, \ldots, 2}^{n-1}, 1)
$$

\footnotetext{
${ }^{9}$ The desired power of the $n$-th voter is implied by the sum condition.

${ }^{10}$ Step size $s$ has to be chosen with care: the number of grid points can be intractably great already for small $n$ if $s$ is too small. But a larger $s$ induces a coarser grid of feasible target vectors. This becomes more and more problematic as $n$ increases because of the corresponding natural decrease of an individual voter's relative power (on average equal to $1 / n)$. Choosing $s=0.03$, for instance, would result in only 297 different grid points (i.e., distinct power distributions with $\beta_{1} \geq \beta_{2} \geq \ldots \geq \beta_{n}$ and $\beta_{i}=k_{i} s$ for $\left.k_{i} \in \mathbb{N}\right)$ for $n=17$ as opposed to 1297 points for $n=8$.
} 


\begin{tabular}{|r|r|ccccc|ccccc|}
\hline & \multicolumn{1}{|c|}{ \#grid } & \multicolumn{6}{|c|}{$d_{1}$-metric } & \multicolumn{5}{|c|}{$d_{\infty}$-metric } \\
$n$ & points & med. & av. & $10 \%$ & $5 \%$ & $1 \%$ & med. & av. & $10 \%$ & $5 \%$ & $1 \%$ \\
\hline 2 & 51 & 0.480 & 0.480 & 0.080 & 0.020 & 0.000 & 0.240 & 0.240 & 0.040 & 0.010 & 0.000 \\
3 & 858 & 0.563 & 0.562 & 0.200 & 0.140 & 0.060 & 0.282 & 0.281 & 0.100 & 0.070 & 0.030 \\
4 & 7519 & 0.427 & 0.512 & 0.207 & 0.160 & 0.087 & 0.210 & 0.251 & 0.087 & 0.067 & 0.037 \\
5 & 41334 & 0.340 & 0.446 & 0.165 & 0.129 & 0.077 & 0.150 & 0.208 & 0.063 & 0.049 & 0.029 \\
6 & 160668 & 0.293 & 0.383 & 0.129 & 0.105 & 0.067 & 0.117 & 0.173 & 0.045 & 0.036 & 0.023 \\
7 & 477213 & 0.240 & 0.330 & 0.102 & 0.081 & 0.053 & 0.093 & 0.146 & 0.033 & 0.026 & 0.016 \\
8 & 1145180 & 0.200 & 0.289 & 0.080 & 0.064 & 0.043 & 0.076 & 0.127 & 0.025 & 0.019 & 0.012 \\
9 & 2320234 & 0.170 & 0.256 & 0.066 & 0.053 & 0.036 & 0.064 & 0.112 & 0.019 & 0.015 & 0.009 \\
10 & 4094767 & 0.147 & 0.230 & 0.056 & 0.045 & 0.031 & 0.055 & 0.101 & 0.016 & 0.012 & 0.008 \\
11 & 6449747 & 0.129 & 0.210 & 0.049 & 0.040 & 0.028 & 0.048 & 0.092 & 0.013 & 0.010 & 0.006 \\
12 & $(100000)$ & 0.031 & 0.036 & 0.020 & 0.018 & 0.014 & 0.008 & 0.010 & 0.004 & 0.004 & 0.003 \\
13 & $(100000)$ & 0.026 & 0.029 & 0.017 & 0.015 & 0.012 & 0.006 & 0.008 & 0.003 & 0.003 & 0.002 \\
14 & $(100000)$ & 0.022 & 0.024 & 0.015 & 0.013 & 0.011 & 0.005 & 0.006 & 0.003 & 0.002 & 0.002 \\
15 & $(100000)$ & 0.019 & 0.021 & 0.013 & 0.012 & 0.010 & 0.004 & 0.005 & 0.002 & 0.002 & 0.002 \\
\hline
\end{tabular}

Table 6: Distribution of absolute deviations for the 50\%-heuristic

\begin{tabular}{|r|r|rcccc|ccccc|}
\hline & \#grid & \multicolumn{6}{|c|}{$d_{1}$-metric } & \multicolumn{5}{|c|}{$d_{\infty}$-metric } \\
$n$ & points & med. & av. & $10 \%$ & $5 \%$ & $1 \%$ & med. & av. & $10 \%$ & $5 \%$ & $1 \%$ \\
\hline 2 & 51 & 0.480 & 0.480 & 0.080 & 0.020 & 0.000 & 0.240 & 0.240 & 0.040 & 0.010 & 0.000 \\
3 & 858 & 0.380 & 0.425 & 0.160 & 0.107 & 0.040 & 0.190 & 0.213 & 0.080 & 0.053 & 0.020 \\
4 & 7519 & 0.340 & 0.366 & 0.160 & 0.120 & 0.060 & 0.145 & 0.170 & 0.065 & 0.050 & 0.025 \\
5 & 41334 & 0.271 & 0.306 & 0.133 & 0.107 & 0.066 & 0.110 & 0.134 & 0.052 & 0.040 & 0.024 \\
6 & 160668 & 0.220 & 0.256 & 0.108 & 0.087 & 0.058 & 0.085 & 0.108 & 0.037 & 0.030 & 0.020 \\
7 & 477213 & 0.180 & 0.216 & 0.084 & 0.069 & 0.046 & 0.065 & 0.089 & 0.026 & 0.021 & 0.014 \\
8 & 1145180 & 0.150 & 0.183 & 0.063 & 0.051 & 0.034 & 0.053 & 0.074 & 0.019 & 0.015 & 0.010 \\
9 & 2320234 & 0.125 & 0.157 & 0.048 & 0.038 & 0.025 & 0.043 & 0.063 & 0.013 & 0.010 & 0.006 \\
10 & 4094767 & 0.104 & 0.137 & 0.036 & 0.028 & 0.018 & 0.035 & 0.055 & 0.010 & 0.007 & 0.004 \\
11 & 6449747 & 0.087 & 0.121 & 0.028 & 0.021 & 0.013 & 0.029 & 0.049 & 0.007 & 0.005 & 0.003 \\
12 & $(100000)$ & 0.017 & 0.021 & 0.009 & 0.008 & 0.006 & 0.004 & 0.005 & 0.002 & 0.002 & 0.001 \\
13 & $(100000)$ & 0.012 & 0.015 & 0.006 & 0.005 & 0.004 & 0.003 & 0.004 & 0.001 & 0.001 & 0.001 \\
14 & $(100000)$ & 0.008 & 0.011 & 0.004 & 0.004 & 0.003 & 0.002 & 0.003 & 0.001 & 0.001 & 0.000 \\
15 & $(100000)$ & 0.005 & 0.008 & 0.003 & 0.003 & 0.002 & 0.001 & 0.002 & 0.001 & 0.000 & 0.000 \\
\hline
\end{tabular}

Table 7: Distribution of absolute deviations for the $q^{\star}$-heuristic 


\begin{tabular}{|r|r|ccccc|ccccc|}
\hline & \multicolumn{1}{|c|}{ \#grid } & \multicolumn{6}{|c|}{$d_{1}$-metric } & \multicolumn{5}{c|}{$d_{\infty}$-metric } \\
$n$ & \multicolumn{1}{|c|}{ points } & med. & av. & $10 \%$ & $5 \%$ & $1 \%$ & med. & av. & $10 \%$ & $5 \%$ & $1 \%$ \\
\hline 2 & 51 & 0.280 & 0.327 & 0.040 & 0.020 & 0.000 & 0.140 & 0.164 & 0.020 & 0.010 & 0.000 \\
3 & 858 & 0.320 & 0.334 & 0.140 & 0.100 & 0.040 & 0.160 & 0.167 & 0.070 & 0.050 & 0.020 \\
4 & 7519 & 0.300 & 0.305 & 0.150 & 0.113 & 0.060 & 0.130 & 0.138 & 0.065 & 0.050 & 0.025 \\
5 & 41334 & 0.247 & 0.261 & 0.132 & 0.101 & 0.060 & 0.100 & 0.109 & 0.050 & 0.040 & 0.023 \\
6 & 160668 & 0.200 & 0.220 & 0.103 & 0.085 & 0.056 & 0.075 & 0.086 & 0.035 & 0.028 & 0.019 \\
7 & 477213 & 0.153 & 0.181 & 0.077 & 0.063 & 0.043 & 0.055 & 0.067 & 0.024 & 0.020 & 0.013 \\
8 & 1145180 & 0.117 & 0.148 & 0.056 & 0.046 & 0.032 & 0.040 & 0.052 & 0.016 & 0.013 & 0.009 \\
9 & 2320234 & 0.093 & 0.125 & 0.042 & 0.034 & 0.024 & 0.030 & 0.043 & 0.012 & 0.009 & 0.006 \\
10 & 4094767 & 0.073 & 0.106 & 0.031 & 0.025 & 0.017 & 0.023 & 0.036 & 0.008 & 0.006 & 0.004 \\
11 & 6449747 & 0.065 & 0.094 & 0.025 & 0.020 & 0.013 & 0.021 & 0.032 & 0.006 & 0.005 & 0.003 \\
12 & $(100000)$ & 0.017 & 0.021 & 0.009 & 0.008 & 0.006 & 0.004 & 0.005 & 0.002 & 0.002 & 0.001 \\
13 & $(100000)$ & 0.012 & 0.015 & 0.006 & 0.006 & 0.004 & 0.003 & 0.004 & 0.001 & 0.001 & 0.001 \\
14 & $(100000)$ & 0.008 & 0.011 & 0.004 & 0.004 & 0.003 & 0.002 & 0.002 & 0.001 & 0.001 & 0.001 \\
15 & $(100000)$ & 0.006 & 0.008 & 0.003 & 0.003 & 0.002 & 0.001 & 0.002 & 0.001 & 0.000 & 0.000 \\
\hline
\end{tabular}

Table 8: Distribution of absolute deviations for the $\bar{q}$-heuristic

for $n \geq 2 .{ }^{11}$ For any quota $q \in I_{1}^{j}:=\frac{1}{2 n-1} \cdot(2 j-1,2 j]$, where $1 \leq j \leq n-1$ and $j \in \mathbb{N}$, the PBI of the smallest constituency is exactly zero and, by symmetry, the PBI of the other constituencies equals $\frac{1}{n-1}$. For the remaining possibilities $q \in I_{2}^{j}:=\frac{1}{2 n-1} \cdot(2 j, 2 j+1]$ where $0 \leq j \leq n-1$, all constituencies have a PBI of $\frac{1}{n}$. Denoting the corresponding weighted games by $v_{1, j}^{n}$ and $v_{2, j}^{n}$ one obtains

$$
\begin{aligned}
& d_{1}\left(v_{1, j}^{n}, \beta^{n}\right)=\frac{2}{2 n-1}, \\
& d_{1}\left(v_{2, j}^{n}, \beta^{n}\right)=\frac{2}{2 n-1} \cdot \frac{n-1}{n}, \\
& d_{\infty}\left(v_{1, j}^{n}, \beta^{n}\right)=\frac{1}{2 n-1}, \text { and } \\
& d_{\infty}\left(v_{2, j}^{n}, \beta^{n}\right)=\frac{1}{2 n-1} \cdot \frac{n-1}{n} .
\end{aligned}
$$

So independently of the quota the $\|\cdot\|_{1}$-error is $\frac{2}{2 n-1}+O\left(n^{-2}\right)$ and the $\|\cdot\|_{\infty}$-error is $\frac{1}{2 n-1}+O\left(n^{-2}\right)$.

The $q^{*}$ and $\bar{q}$-heuristics prescribe quotas of

$$
\begin{aligned}
& \bar{q}=\frac{1}{2}+\frac{1}{\sqrt{\pi n}}, \text { and } \\
& q^{*}=\frac{1}{2}+\frac{\sqrt{4 n-3}}{4 n-2},
\end{aligned}
$$

respectively. They and $q^{\circ}=50 \%$ fall into $I_{1}^{j}$ and $I_{2}^{j}$ for infinitely many $n$. Thus, all three rules render the smallest constituency a null player infinitely many times as $n \rightarrow \infty$, just as it happened to Luxembourg in the EEC Council between 1958 and 1973.

In contrast, there is always a simple game whose PBI attains $\beta^{n}$ exactly for $6 \leq n \leq 13$. And we conjecture that this remains true for $n \geq 14$. Approximation results for complete simple games and the heuristic choice of $w=\beta^{n}$ with an "optimal" quota $q$ that leads to $v_{2, j}^{n}$ (abbreviated as q-heuristic) are summarized in Tables 9 and 10. Since the unavoidable error in the class of simple games $\mathcal{S}$ (and hence of finite intersections of weighted games $v \in \mathcal{W}$ ) is zero for $6 \leq n \leq 13$ and presumably beyond,

\footnotetext{
${ }^{11}$ The construction is inspired by a sequence of weighted voting games to which the Penrose limit theorem (see fn. 3 ) does not apply.
} 


\begin{tabular}{|r|c|c|c|cc|}
\hline & $v^{*} \in \mathcal{S}$ & $v^{* *} \in \mathcal{C}$ & $v^{* * *} \in \mathcal{W}$ & \multicolumn{2}{|c|}{$q$-heuristic } \\
$n$ & $d_{1}$ & $d_{1}$ & $d_{1}$ & $d_{1}$ & $\mathcal{C}$-error \\
\hline 2 & 0.333333 & 0.333333 & 0.333333 & 0.333333 & 0.000000 \\
3 & 0.266667 & 0.266667 & 0.266667 & 0.266667 & 0.000000 \\
4 & 0.214286 & 0.214286 & 0.214286 & 0.214286 & 0.000000 \\
5 & 0.038647 & 0.158730 & 0.158730 & 0.177778 & 0.120000 \\
6 & 0.000000 & 0.113636 & 0.113636 & 0.151515 & 0.333333 \\
7 & 0.000000 & 0.085470 & 0.085470 & 0.131868 & 0.542857 \\
8 & 0.000000 & 0.066667 & 0.066667 & 0.116667 & 0.750000 \\
9 & 0.000000 & 0.064171 & 0.064171 & 0.104575 & 0.629630 \\
10 & 0.000000 & 0.061042 & 0.061042 & 0.094737 & 0.552000 \\
11 & 0.000000 & 0.052158 & 0.052158 & 0.086580 & 0.659944 \\
12 & 0.000000 & 0.047254 & 0.047254 & 0.079710 & 0.686856 \\
13 & 0.000000 & $0.042353^{\dagger}$ & $0.044483^{\dagger}$ & 0.073846 & 0.743590 \\
\hline
\end{tabular}

Table 9: Deviations from $\beta^{n}$ in the $d_{1}$-metric (analytical example)

we consider the $\mathcal{C}$-error in order to evaluate the relative performance of the $q$-heuristic. Interestingly, the $\mathcal{C}$-error in the $d_{1}$-metric seems to converge to a constant while it seems to grow without bound for the $d_{\infty}$-metric.

\begin{tabular}{|r|c|c|c|cc|}
\hline & $v^{*} \in \mathcal{S}$ & $v^{* *} \in \mathcal{C}$ & $v^{* * *} \in \mathcal{W}$ & \multicolumn{2}{|c|}{$q$-heuristic } \\
$n$ & $d_{\infty}$ & $d_{\infty}$ & $d_{\infty}$ & $d_{\infty}$ & $\mathcal{C}$-error \\
\hline 2 & 0.166667 & 0.166667 & 0.166667 & 0.166667 & 0.000000 \\
3 & 0.133333 & 0.133333 & 0.133333 & 0.133333 & 0.000000 \\
4 & 0.107143 & 0.107143 & 0.107143 & 0.107143 & 0.000000 \\
5 & 0.019324 & 0.050505 & 0.050505 & 0.088889 & 0.760000 \\
6 & 0.000000 & 0.034759 & 0.034759 & 0.075758 & 1.179487 \\
7 & 0.000000 & 0.022624 & 0.022624 & 0.065934 & 1.914286 \\
8 & 0.000000 & 0.015686 & 0.015686 & 0.058333 & 2.718750 \\
9 & 0.000000 & 0.014199 & 0.014199 & 0.052288 & 2.682540 \\
10 & 0.000000 & 0.008772 & 0.008772 & 0.047368 & 4.400000 \\
11 & 0.000000 & 0.008282 & 0.008282 & 0.043290 & 4.227273 \\
12 & 0.000000 & 0.007688 & 0.007688 & 0.039855 & 4.183908 \\
13 & 0.000000 & $0.005373^{\dagger}$ & $0.007083^{\dagger}$ & 0.036923 & 5.871952 \\
\hline
\end{tabular}

Table 10: Deviations from $\beta^{n}$ in the $d_{\infty}$-metric (analytical example)

\section{Conclusion}

The computations which we have reported in Section 5.1 confirm that if one wants to implement the Penrose square root rule for population data from today's European Union, the $q^{*}$-heuristic of Słomczyński and Życzkowski and, to a lesser extent, the even simpler $\bar{q}$-heuristic perform very well in absolute terms. That is, the distance between a (normalized) square root target distribution $\beta$ and the PBI $B\left(q^{*}, \beta\right)$ is close to zero. However, the considered heuristics can still be very far from the globally optimal solution to the inverse problem in relative terms. This finding applies even when only weighted voting games are allowed as feasible solutions. And it is not restricted to small voting bodies, but holds for the current number of EU members $n=27$.

The extensive computations reported in Section 5.2 confirm this observation. They provide the 
first systematic evaluation of the unavoidable deviations between arbitrary target PBI power vectors and those that are actually implementable for voting bodies with up to $n=15$ members. Numbers such as the ones reported in Table 5 can potentially be useful in order to improve termination criteria for local search algorithms (e.g., Leech 2002, 2003), which have been used in applied studies. If, say, a locally optimal candidate solution for an inverse problem with $n=11$ voters has a $d_{1}$-deviation from the desired vector $\beta$ greater than 0.020, then Table 5 indicates that the odds of further improvements in the class of weighted voting games are 50:50 and search presumably should continue in a different part of the game space. If, however, the deviation is smaller than 0.009, then the odds are rather 1:99; termination might then make sense.

That desired PBI distributions which concentrate a major share of relative power amongst a few voters pose problems for the considered heuristics is not surprising. After all, the derivation of $q^{*}$ by Słomczyński and Życzkowski (2007) supposed a technical condition (see fn. 4) from which one can conclude $w_{j}=\beta_{j} \in O(1 / \sqrt{n})$, i.e., the PBI of a single voter should approach zero as least as fast as $1 / \sqrt{n}$. It is much less obvious, however, that (i) it is not sufficient to have a target vector $\beta$ without "outliers" in order to obtain a heuristic solution that is good relative to the exact one and (ii) the relative errors may get larger rather than smaller as $n$ increases. This emerged from the extensive numerical computations reported in Sections 5.1-5.2 and has also been demonstrated for a specific analytical example in Section 5.3. One might, therefore, summarize our findings as justifying and potentially even calling for case-specific optimization rather than the application of a generally rather good heuristic - not only for small but even for large voting bodies.

\section{References}

Alon, N. and P. H. Edelman (2010). The inverse Banzhaf problem. Social Choice and Welfare 34(3), $371-377$.

Aziz, H., M. Paterson, and D. Leech (2007). Efficient algorithm for designing weighted voting games. In Multitopic Conference, 200\%. INMIC 200\%. IEEE International, pp. 1-6. Available at http://eprints.dcs.warwick.ac.uk/1547/.

Banzhaf, J. F. (1965). Weighted voting doesn't work: A mathematical analysis. Rutgers Law Review $19(2), 317-343$.

Carreras, F. and J. Freixas (1996). Complete simple games. Mathematical Social Sciences 32(2), 139155.

Chang, P.-L., V. C. Chua, and M. Machover (2006). L S Penrose's limit theorem: Tests by simulation. Mathematical Social Sciences 51(1), 90-106.

Del̆neko, V. G. and G. J. Woeginger (2006). On the dimension of simple monotonic games. European Journal of Operational Research 170(1), 315-318.

Dubey, P. and L. Shapley (1979). Mathematical properties of the Banzhaf power index. Mathematics of Operations Research 4(2), 99-131.

Fatima, S., M. Wooldridge, and N. Jennings (2008). An anytime approximation method for the inverse Shapley value problem. In Proceedings of the 7th International Conference on Autonomous Agents and Multi-Agent Systems, Estoril, Portugal, pp. 935-942. Available at http://eprints.ecs.soton.ac.uk/15131.

Felsenthal, D. and M. Machover (1998). The Measurement of Voting Power - Theory and Practice, Problems and Paradoxes. Cheltenham: Edward Elgar.

Freixas, J. and X. Molinero (2009). On the existence of a minimum integer representation for weighted voting systems. Annals of Operations Research 166, 243-260.

Grilli di Cortona, P., C. Manzi, A. Pennisi, F. Ricca, and B. Simeone (1999). Evaluation and optimization of electoral systems. SIAM Monographs on Discrete Mathematics and Applications. Philadelphia, PA: Society for Industrial and Applied Mathematics (SIAM). 
Isbell, J. R. (1956). A class of majority games. Quarterly Journal of Mathematics 7(1), 183-187.

Kaniovski, S. (2008). The exact bias of the Banzhaf measure of power when votes are neither equiprobable nor independent. Social Choice and Welfare 31(2), 281-300.

Keijzer, B. d. (2009). On the design and synthesis of voting games. Master's thesis, Delft University of Technology.

Keijzer, B. d., T. Klos, and Y. Zhang (2010). Enumeration and exact design of weighted voting games. In Proceedings of the 9th International Conference on Autonomous Agents and Multiagent Systems, Volume 1, pp. 391-398.

Kirsch, W., W. Słomczyński, and K. Życzkowski (2007). Getting the votes right. European Voice (3-9 May), 12.

Kurth, M. (2008). Square root voting in the Council of the European Union: Rounding effects and the Jagiellonian Compromise. Available at http://arxiv.org/abs/0712.2699.

Kurz, S. (2012a). On minimum sum representations for weighted voting games. Annals of Operations Research (forthcoming). DOI: 10.1007/s10479-012-1108-3.

Kurz, S. (2012b). On the inverse power index problem. Optimization (forthcoming). DOI:10.1080/02331934.2011.587008.

Kurz, S., N. Maaser, and S. Napel (2012). On the egalitarian weights of nations. mimeo, University of Bayreuth.

Laruelle, A. and F. Valenciano (2008). Voting and Collective Decision-Making. Cambridge: Cambridge University Press.

Leech, D. (2002). Designing the voting system for the EU Council of Ministers. Public Choice 113(3-4), 437-464.

Leech, D. (2003). Power indices as an aid to institutional design: The generalised apportionment problem. In M. J. Holler, H. Kliemt, D. Schmidtchen, and M. E. Streit (Eds.), Jahrbuch Für Neue Politische Ökonomie, Volume 22. Tübingen: Mohr Siebeck.

Lindner, I. and M. Machover (2004). L. S. Penrose's limit theorem: Proof of some special cases. Mathematical Social Sciences 47(1), 37-49.

Lindner, I. and G. Owen (2007). Cases where the Penrose limit theorem does not hold. Mathematical Social Sciences 53(3), 232-238.

Pennisi, A., F. Ricca, P. Serafini, and B. Simeone (2007). Amending and enhancing electoral laws through mixed integer programming in the case of Italy. In E. Yashin (Ed.), Proceedings of the 8th International Conference on Economic Modernization and Social Development. HSE, Moscow.

Penrose, L. S. (1946). The elementary statistics of majority voting. Journal of the Royal Statistical Society $109(1), 53-57$.

Penrose, L. S. (1952). On the Objective Study of Crowd Behaviour. London: H. K. Lewis \& Co.

Ricca, F., A. Scozzari, P. Serafini, and B. Simeone (2012). Error minimization methods in biproportional apportionment. TOP (forthcoming). Available at http://sole.dimi.uniud.it/ paolo.serafini/TOPMINERR.pdf.

Shapley, L. S. and M. Shubik (1954). A method for evaluating the distribution of power in a committee system. American Political Science Review 48(3), 787-792.

Słomczyński, W. and K. Życzkowski (2006). Penrose voting system and optimal quota. Acta Physica Polonica B 37, 3133-3143.

Słomczyński, W. and K. Życzkowski (2007). From a toy model to the double square root system. Homo Oeconomicus $24(3 / 4), 381-399$.

Słomczyński, W. and K. Życzkowski (2011). Square root voting system, optimal threshold and $\pi$. Available a thttp://arxiv.org/abs/1104.5213.

Taylor, A. D. and W. S. Zwicker (1999). Simple Games. Princeton, NJ: Princeton University Press. 


\section{Appendix: ILP formulation for the inverse Penrose-Banzhaf index problem}

The following ILP formulation considers the inverse problem on the class of simple games $\mathcal{S}$ and for the $d_{1}$-metric. Adaptations to $\mathcal{C}$ or $\mathcal{W}$ and $d_{1}^{\prime}$ or $d_{\infty}$ involve further variables and (modified) constraints, but are otherwise very similar:

$$
\begin{array}{ll}
x_{S} \in\{0,1\} & \forall S \subseteq N, \\
x_{S} \leq x_{T} & \forall S \subseteq T \subseteq N, \\
x_{\emptyset}=0 & \\
x_{N}=1 & \\
y_{i, S} \in\{0,1\} & \forall 1 \leq i \leq n, S \subseteq N \backslash\{i\}, \\
y_{i, S}=x_{S \cup\{i\}}-x_{S} & \forall 1 \leq i \leq n, S \subseteq N \backslash\{i\}, \\
s_{i} \geq 0 & \forall 1 \leq i \leq n, \\
s_{i}=\sum_{S \subseteq N \backslash\{i\}} y_{i, S} & \\
s=\sum_{i=1}^{n} s_{i}, & \forall 1 \leq i \leq n, \\
\delta_{i} \geq 0 & \forall 1 \leq i \leq n, \\
\delta_{i} \geq s_{i}-\beta_{i} \cdot s & \forall 1 \leq i \leq n, \\
\delta_{i} \geq-s_{i}+\beta_{i} \cdot s & \\
\sum_{i=1}^{n} \delta_{i} \leq \alpha \cdot s . &
\end{array}
$$

The binary variables $x_{S}$ define a Boolean function $v$ via $v(S)=x_{S}$; inequalities (3)-(6) ensure that they represent a simple game. The binary auxiliary variables $y_{i, S}=x_{S \cup\{i\}}-x_{S}$ which are introduced in (7)-(8) for all $i \in N$ and $\emptyset \subseteq S \subseteq N \backslash\{i\}$ satisfy $y_{i, S}=1$ if and only if coalition $S$ is a swing for voter $i$. With this the number of swings $s_{i}$ for each player $i$ is determined in equality (10). Since the PBI for voter $i$ is given by $B_{i}(v)=s_{i} / \sum_{j=1}^{n} s_{j}$, the objective is to minimize the $d_{1}$-distance

$$
\sum_{i=1}^{n}\left|\frac{s_{i}}{\sum_{j=1}^{n} s_{j}}-\beta_{i}\right| .
$$

Unfortunately, the quotient cannot be linearized. We, therefore, introduce $s=\sum_{i=1}^{n} s_{i}$ in inequality (11) and capture $\delta_{i} \geq\left|s_{i}-\beta_{i} \cdot s\right|$ by inequalities (13) and (14). ${ }^{12}$ Instead of directly minimizing the sum of all $\delta_{i}$ we introduce the constraint (15) for a constant $\alpha \in[0,2]$. Here, $\alpha=2$ reflects the supremum of $d_{1}$-distances between elements of $\Delta(n-1)$, and $\alpha=0$ corresponds to identity of $B(v)$ and desired vector $\beta$.

It remains to note that if the ILP (3)-(15) has a solution, then the corresponding simple game $v$ approximates the desired power distribution $\beta$ with an error of at most $\alpha$ in the $\|\cdot\|_{1}$-norm. Otherwise, no such approximation is possible. We can hence minimize the deviation by performing bisections on $\alpha$. Since $s$ lies between $n$ and $m\left(\begin{array}{c}n \\ m\end{array}\right)<n 2^{n}$ where $m=\left\lfloor\frac{n}{2}\right\rfloor+1$ (see, e.g., Felsenthal and Machover 1998, sec. 3.3) two distinct PBI vectors differ, both in the $d_{1}$ - and the $d_{\infty}$-metric, by at least $\left(\frac{1}{n 2^{n}}\right)^{2}$. We hence only need $O(n)$ bisections on $\alpha$. The computations were been carried out using the Gurobi 4.6 and the IBM ILOG CPLEX 12.4 software packages.

\footnotetext{
${ }^{12}$ Interestingly, one can easily linearize the analogous inverse problem for the Shapley-Shubik power index (SSI). So even though the PBI is easier to compute than the SSI, the corresponding inverse problem is slightly more difficult.
} 